\title{
APPLICATIONS OF THE EXTREMUM PRINCIPLES FOR NON-NEWTONIAN FLUIDS*
}

NAOYA YOSHIOKA AND KITARO ADACHI

Department of Chemical Engineering, Kyoto University, Kyoto, Japan

\begin{abstract}
The variational principles for creeping flow of non-Newtonian fluids are utilized with considerable success when the common value of the functionals in the actual state is connected with that of an important unknown. Two lines are examined. The one is to extend the results given by Hill for a Newtonian fluid to those for a non-Newtonian fluid, and the other is to exfend the idea used by Haddow for visco-plastic torsion of a prismatic bar to the general case.
\end{abstract}

\section{Introduction}

In a previous paper $^{9}$ the authors formulated extremum principles for a non-Newtonian fluid. In this paper, methods of estimating important physical quantities by using the inequalities derived from the extremum principles are described. This problem involves selection of trial functions.

It was probably Lord Rayleigh who first drew attention to the formal similarity between the field equations for an elastic solid in equilibrium and those for Newtonian viscous fluid in quasi-static flow. Hill $^{2}$ also emphasized the mutual utilization of the exact and partial correspondences between the field equations.

Approximation is made by relaxation of constraints such as field equations, constitutive equations, boundary conditions and geometric shapes of boundaries. The exact solution of the approximate boundary value problem obtained in such a way is regarded as an approximate solution of the original and exact boundary value problem.

Admissible functions related with extremum principles are selected from such approximate solutions as described above. In this paper, not mathematical conditions but rather physical or geometric ones are relaxed: that is, the fluid in a flow region $V$ or the boundary is altered.

Under certain types of boundary conditions and constitutive equations, the common value of the functionals related with extremum principles in the actual state is directly connected with that of an important unknown: for example, the drag on a moving body or its velocity. In such cases an approximate value of the important unknown can be obtained by bracketing it between upper and lower bounds corresponding to the admissible functions

* Received on October 31, 1970 used there. But under the other situations the common value is indirectly connected to or independent of the value of an important unknown.

The former method has been exploited with considerable success for problems of the deformation and flow of elastic, elastic-plastic and rigid-plastic solids. Furthermore, it was also applied to the pipeline flow of a Newtonian liquid by Ito $^{4}$, to that of an Ostwaldde Waele fluid by Schechters), and to the flow of a non-Newtonian fluid past a sphere by Tomita ${ }^{7)}$ and by Slattery ${ }^{6}$.

Two ideas on how to look for the admissible functions were suggested by Hill's $\mathrm{s}^{3}$ and Haddow's work ${ }^{1}$.

Hill $^{3}$ ) estimated the drag on a body moving slowly through a fluid by the drag on another body by which the original body would by replaced, and he also obtained such a qualitative but important conclusion as that the apparent viscosity of a suspension is a monotonically increasing function of the volume concentration of solute. Haddow ${ }^{1}$ derived upper and lower bounds of the torque required to twist a prismatic visco-plastic bar by using the solution of velocity field for the same boundary value problem of a Newtonian fluid and the solution of stress field for that of a rigid perfectly plastic solid.

The purpose of this paper is to indicate how to look for more suitable admissible functions connected with the extremum principles in order to obtain close estimates of the drag or velocity for flows of a nonNewtonian fluid.

\section{Basic Matters}

An isotropic and incompressible non-Newtonian fluid is treated. The constitutive equations are

$$
\tau_{i j}=2 \eta\left(\Pi_{s}\right) \varepsilon_{i j} \text { or } \varepsilon_{i j}=2 \hat{\eta}\left(\Pi_{\tau}\right) \tau_{i j} ; \Pi_{\tau} \leqslant k
$$

and the work function and the complementary one are 


$$
\Gamma\left(\Pi_{\ell}\right)=\int \tau_{i j} d \varepsilon_{i j} \quad \text { and } \quad \hat{\Gamma}\left(\Pi_{\tau}\right)=\int \varepsilon_{i j} d \tau_{i j}
$$

respectively, where

$$
\Pi_{\varepsilon}^{2}=\varepsilon_{i j} \varepsilon_{i j}, \quad \mathrm{II}_{\tau}^{2}=\tau_{i j} \tau_{i j}
$$

These constitutive relations are valid throughout the region $V$ except at the surface of velocity discontinuity across which the velocity is tangentially discontinuous and along which the stress is a pure shear $k$. No shearing stress can exceed $k$ numerically in $V$. Then, the Newtonian fluid, the generalized Newtonian fluid and the Bingham fluid are limiting cases of the fluid considered here. Indeed, Eq.(1) characterizes a Newtonian fluid when $k \rightarrow \infty$ and $\eta\left(\Pi_{\varepsilon}\right)=$ a positive constant, a generalized Newtonian fluid when $k \rightarrow \infty$ and $\eta\left(\Pi_{\varepsilon}\right)=$ a positive-valued function of $\Pi_{s}$, and a Bingham fluid when $k \rightarrow \infty$ and $\eta\left(\Pi_{s}\right)=\mu_{B}+\tau_{y} / \Pi_{\varepsilon}$.

In a previous paper ${ }^{9}$, the authors have presented the following two extremum principles for the fluid.

The minimum principle concerned with velocity fields states that the actual velocity field minimizes the functional

$$
\begin{aligned}
H\left(\Pi_{i}^{+}\right)= & \iiint\left[\Gamma\left(\Pi_{\dot{c}}^{+}\right)-\rho f_{i} \mathscr{V}_{i}^{+}\right] d V \\
& +\iint T_{d} \mathscr{Y}_{d}^{+} d S_{d}^{+}-\iint T_{i} \mathscr{Y}_{i}^{+} d S_{t}
\end{aligned}
$$

where $\mathscr{Y}_{i}^{+}$is any kinematically admissible velocity field that is piecewise continuous with piecewise continuous first derivatives and that satisfies the velocity boundary conditions on $S_{v}$ and the condition of incompressibility.

The maximum principle concerned with stress fields states that the actual stress field maximizes the functional

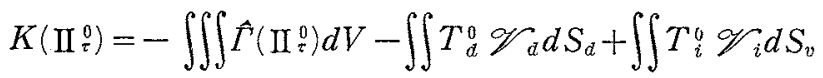

where $\sigma_{i j}^{0}$ is statically any admissible stress field that is continuous with piecewise continuous first derivatives and that satisfies the stress boundary conditions on $S_{t}$ and the equations of motion with neglected inertia terms.

In addition to these principles, the principle of virtual work will be used repeatedly in the followig analysis, and is expressed by the equation

$$
\begin{aligned}
\iiint \sigma_{i j}^{0} \varepsilon_{i j}^{+} d V+\iint T_{d}^{0} \mathscr{Y}_{d}^{+} d S_{d}^{+}= & \iiint \rho f_{i} \mathscr{Y}_{i}^{+} d V \\
& +\iint T_{i}^{0} \mathscr{Y}_{i}^{+} d S
\end{aligned}
$$

Now, it is supposed that a rigid body moves in a fluid which is completely contained within rigid boundaries at rest or extends to infinity where the fluid is at rest, or that a fluid is confined by two rigid boundaries and one moves while the other is at rest, or that a fluid flows through a duct. Here, unbounded two-dimensional flows of pure viscous fluids produced by pure translation are excluded, since the only quasistatic motion possible to the fluid is a trivial rigidbody one. The flow under consideration is steady laminar with no body forces and no velocity discontinuity. In consequence, the Eq.(6) is rewritten as follows

$$
\begin{aligned}
& \iiint \sigma_{i j}^{0} \varepsilon_{i j}^{+} d V=\iint T_{i}^{0 \mathscr{Y}}{ }_{i}^{+} d S \\
& =F^{0} U^{+}+T^{0} \Omega^{+} \text {(or }=\Delta P^{0} \cdot U^{+} S \text { ) }
\end{aligned}
$$

when the motion of the body is specified by the velocity $U^{+}$of some point 0 of it and by the spin $\Omega^{+}$ about the point ( $U^{+}$is the mean velocity of the fluid in the duct if a pipeline flow is considered), and the tractions on the body are equivalent to a force $F^{0}$ and a couple $T^{0}$ about $0\left(\Delta P^{0}\right.$ is the pressure-drop in the duct). In particular, if $\sigma_{i j}^{0}$ and $\varepsilon_{i j}^{+}$are of the actual flow, the left-hand side of Eq.(7) shows the rate of dissipation of energy and is denoted by $E$.

$$
E=F U+T \Omega(\text { or }=\Delta P \cdot U S)
$$

In this paper, two types of boundary conditions are considered: (a) zero prescribed surface tractions on $S_{t}$, and (b) zero prescribed velocities on $S_{v}$.

For no body forces and no velocity discontinuity, the following inequalities are obtained from the extremum principles

$$
H\left(\Pi_{\varepsilon}^{+}\right) \geqslant H\left(\Pi_{e}\right)=K\left(\Pi_{\varepsilon}\right) \geqslant K\left(\Pi_{\tau}^{0}\right)
$$

where for the case (a)

$$
\begin{aligned}
H\left(\Pi_{\varepsilon}^{+}\right) & =\iiint \Gamma\left(\Pi_{s}^{+}\right) d V \\
K\left(\Pi_{\bullet}^{0}\right) & =-\iiint \hat{\Gamma}\left(\Pi_{\tau}^{0}\right) d V+\iint T_{i}^{0} \mathscr{Y}_{i} d S_{v}
\end{aligned}
$$

and for the case (b)

$$
\begin{aligned}
H\left(\Pi_{s}^{+}\right) & =\iiint \Gamma\left(\Pi_{c}^{+}\right) d V-\iint T_{i} \mathscr{V}_{i}^{+} d S_{t} \\
K\left(\Pi_{\tau}^{0}\right) & =-\iiint \hat{\Gamma}\left(\Pi_{\tau}^{+}\right) d V
\end{aligned}
$$

\section{An Extension of Extremum Principles (I)}

An approximate value of the important unknown $(F U+T \Omega)$ can be obtained here by bracketing it between upper and lower bounds corresponding to adopted admissible functions. The method of evaluating $(F U+T \Omega)$ developed here can be applied only to the flow of a power law fluid. As the first variations of the functionals vanish in the actual state and the common value of the functionals in the actual state is directly connected with that of an important unknown $(F U+T \Omega)$, a close approximation to $(F U+T \Omega)$ can usually be obtained from comparatively simple admissible functions.

It is assumed that $H\left(\mathrm{II}_{\varepsilon}\right)$ is a function only of the total rate of dissipation of energy $E$. This condition requires that $\Gamma\left(\Pi_{\varepsilon}\right)$ and $\hat{\Gamma}\left(\Pi_{t}\right)$ must be proportional to $\sigma_{i j} \varepsilon_{i j}$, so $\sigma_{i j} d \varepsilon_{i j} \propto \varepsilon_{i j} d \sigma_{i j}$. In consequence the following relation is obtained under the above assumption

$$
\mathrm{II}_{\tau}=2 \mu_{p} \Pi_{\varepsilon}^{n}
$$

where $\mu_{p}$ and $n$ are material constants. This constitutive equation is satisfied only by an Ostwaldde Waele fluid. Then, from Eqs.(2), (14) and the total rate of dissipation energy $E$,

$\iiint \Gamma\left(\Pi_{e}^{+}\right) d V=\frac{1}{n+1} E^{+}, \iiint \hat{\Gamma}\left(\Pi_{-}^{0}\right) d V=\frac{n}{n+1} E^{0}$ 
Here the inequalities (9) are used to evaluate the actual rate of work of the forces needed to move the body in the prescribed way. Substituting Eqs.(10) to (13) into Eq.(9) and using Eqs.(7), (8) and (15), one obtains for the respective case

(a) $E^{+} \geqslant F U+T \Omega \geqslant-n E^{0}+(n+1)\left(F^{0} U+T^{0} \Omega\right)$

(b) $E^{0} \geqslant F U+T \Omega \geqslant \frac{n+1}{n}\left(F U^{+}+T \Omega^{+}\right)-\frac{1}{n} E^{+}$

$$
(n \neq 0)
$$

In particular, if the body is simply translated $(\Omega=0)$ the drag $F$ or the velocity $U$ is evaluated, while if the body is simply rotated $(U=0)$ the torque $T$ or the spin $\Omega$ is evaluated. Furthermore, if the fluid flows through a duct the pressure drop $\Delta P$ or the mean velocity $U$ is evaluated.

Some important deductions similar to Hill's results can be made from Eqs.(16) and (17):

1) If a body with surface $S_{2}$ could be completely contained within a body with surface $S_{1}$, then the drag $F_{1}$ on $S_{1}$ is greater than or equal to the drag $F_{2}$ on $S_{2}$ for case (a) [the velocity of translation and the external boundary of the fluid are the same], or the translational velocity $U_{1}$ of a body with $S_{1}$ is smaller than or equal to the translational velocity $U_{2}$ of another body with $S_{2}$ for case (b) [the drag on any body in pure translation and the external boundary of the fluid are the same]. For the actual flow for $S_{1}$, together with a pure translation in the space $S_{1}-S_{2}$, is an admissible velocity field $\mathscr{V}^{+}$in respect of $S_{2}$. Hence the former is proved by Eq. (16), and the latter can be proved by Eq.(17) in a similar way.

2) If a cylindrical duct with cross section $S_{2}$ and length $L$ could be completely containd within a cylindrical duct with cross section $S_{1}$ and length $L$, the pressure force $\Delta P_{1} S_{1}$ in the latter is smaller than or equal to the pressure force $\Delta P_{2} S_{2}$ in the former for case (a) [the mean velocity and the no-slip condition on the duct wall are the same], or the mean velocity $U_{1}$ in the latter is greater than or equal to the mean velocity $U_{1}$ in the former for case (b) [the pressure force and no-slip condition on the duct wall are the same]. For the actual flow for $S_{2}$, together with fluid at rest in the space $S_{1}-S_{2}$, is an admissible velocity field $\mathscr{V}^{+}$in respect of $S_{1}$. Hence

$$
\begin{aligned}
& \Delta P_{1} S_{1} U \leqslant E^{+}=E_{2}=\Delta P_{2} S_{2} U \\
\therefore \quad & \Delta P_{1} S_{1} \leqslant \Delta P_{2} S_{2}
\end{aligned}
$$

and the latter case can be proved by Eq.(17) in a similar way.

3) The drag $F_{1}$ on a given body in translational motion through a fluid in a stationary rigid container $C_{1}$ is greater than or equal to the drag $F_{2}$ in any container $C_{2}$ which would completely enclose $C_{1}$ for case (a) [the body and its velocity are the same], or the velocity $U_{1}$ in the container $C_{1}$ is smaller than or equal to the velocity $U_{2}$ in the container $C_{2}$ for case (b) [the body and its drag are the same]. For the actual flow within $C_{1}$, together with fluid at rest in the space $C_{2}-C_{1}$, is an admissible field $\mathscr{V}^{+}$in respect of $C_{2}$. Hence, using Eq.(16) for case (a) and Eq.(17) for case (b), one obtains the results;

$$
\begin{aligned}
& F_{2} U \leqslant E^{+}=E_{1}=F_{1} U \\
\therefore & F_{2} \leqslant F_{1} \\
& F U_{2} \geqslant(n+1) F U^{+} / n-E^{+} / n=F U_{1} \\
\therefore & U_{2} \geqslant U_{1}
\end{aligned}
$$

4) The drag on a body $S$ tends to be increased for case (a), or the velocity of the body tends to be decreased for case (b), by the presence of other bodies $S_{1}, S_{2}, \cdots, S_{n}$ which are either fixed in position or free to move without restraint.

5) The apparent viscosity of a non-Newtonian fluid containing a solid suspension is a monotonically increasing function of the volume concentration of solute.

\section{Another Extension of Extremum Principles (II)}

An approximate value of the important unknown $(F U+T \Omega)$ can be obtained by bracketing it between upper and lower bounds corresponding to adopted admissible functions in the extremum principles as before. The method of evaluating $(F U+T \Omega)$ developed here can be applied to the flows of a few nonNewtonian fluids, where a specified constraint must be imposed on the admissible functions to be used. Moreover, since the common value of the functionals in the actual state is not directly connected with that of an important unknown $(F U+T \Omega)$ even though the first variations of the functionals vanish in the actual state, it may not be so easy to obtain a close approximation to the actual value of $(F U+T \Omega)$ from the admissible functions adopted in the related flow problem.

To obtain the desired inequalities bracketing an important unknown from the inequalities bracketing the values of the functional in the extremum principles, several fluids with the following work functions and complementary functions derived from the originally related fluid with $\Gamma\left(\Pi_{s}\right)$ and $\hat{\Gamma}\left(\Pi_{t}\right)$ are utilized:

$$
\begin{aligned}
& \Gamma_{*}\left(\Pi_{\varepsilon}\right) \equiv \hat{\Gamma}\left(\Pi_{\tau}\right) ! \Pi_{\tau}=2 \eta\left(\Pi_{e}\right) \Pi_{\varepsilon} \\
& \hat{\Gamma}_{\mathrm{o}}\left(\Pi_{\tau}\right) \equiv \Gamma\left(\Pi_{\varepsilon}\right) \mid \Pi_{\varepsilon}=2 \hat{\eta}\left(\Pi_{\tau}\right) \Pi_{\tau} \\
& \Gamma_{\Delta}\left(\Pi_{\varepsilon}\right) \equiv \alpha \Gamma\left(\Pi_{\varepsilon}\right)+\beta \Gamma_{*}\left(\Pi_{\varepsilon}\right) \\
& \hat{\Gamma}_{\square}\left(\Pi_{\tau}\right) \equiv \delta \hat{\Gamma}_{o}\left(\Pi_{\tau}\right)+\xi \hat{\Gamma}\left(\Pi_{\tau}\right)
\end{aligned}
$$

where $\alpha, \beta, \delta$ and $\xi$ are suitable constants which must satisfy the following relations for any $\mathrm{II}_{\varepsilon}$ and $\mathrm{II}_{\tau}$ :

$$
\begin{aligned}
& \alpha \Gamma\left(\Pi_{s}\right)+\beta \Gamma_{*}\left(\Pi_{\varepsilon}\right) \geqslant 0 \\
& \delta \hat{\Gamma}_{\mathrm{o}}\left(\Pi_{\tau}\right)+\xi \hat{\Gamma}\left(\Pi_{\tau}\right) \geqslant 0
\end{aligned}
$$

[A] For case (a), the extremum principles described by Eqs.(10) and (11) are used repeatedly in the following way for the fluid given above. That is,

$$
\begin{gathered}
\iiint \Gamma\left(\Pi_{\varepsilon}^{\mathrm{V}}\right) d V \geqslant \iiint \Gamma\left(\Pi_{*}\right) d V \\
-\iiint \hat{\Gamma}\left(\Pi_{\tau}\right) d V+(F U+T \Omega) \geqslant-\iiint \hat{\Gamma}\left(\Pi_{\tau}^{V}\right) d V \\
+\left(F^{\mathrm{V}} U+T^{\mathrm{V}} \Omega\right) \\
\iiint \Gamma_{*}\left(\Pi_{*}\right) d V \geqslant \iiint \Gamma_{*}\left(\Pi_{*}^{*}\right) d V \\
-\iiint \hat{\Gamma}_{\circ}\left(\Pi_{\tau}^{\circ}\right) d V+\left(F^{\circ} U+T^{\circ} \Omega\right)
\end{gathered}
$$




$$
\begin{array}{r}
\geqslant-\iiint \hat{\Gamma}_{\mathrm{o}}\left(\Pi_{\tau}\right) d V+(F U+T \Omega) \\
\iiint \Gamma_{\triangle}\left(\Pi_{\odot}\right) d V \geqslant \iiint \Gamma_{\triangle}\left(\Pi_{s}^{\triangle}\right) d V \\
-\iiint \hat{\Gamma}_{\square}\left(\Pi_{\tau}^{\square}\right) d V+\left(F \square U+T^{\square} \Omega\right) \\
\geqslant-\iiint \hat{\Gamma}_{\square}\left(\Pi_{\tau}\right) d V+(F U+T \Omega)
\end{array}
$$

Then the following relations are obtained from them: (1) when $\alpha, \beta \geqslant \gamma$ and $\delta, \xi \geqslant \lambda$,

$$
\begin{aligned}
& \beta(F U+T \Omega) \geqslant \iiint \Gamma_{\triangle}\left(\Pi_{s}^{\triangle}\right) d V-(\alpha-\gamma) \iiint \Gamma\left(\Pi_{s}^{\mathrm{V}}\right) d V \\
& -(\beta-\gamma)\left[\iiint \hat{\Gamma}\left(\amalg_{\tau}^{\mathrm{V}}\right) d V-\left(F^{\mathrm{V}} U+T^{\mathrm{V}} \Omega\right)\right] \\
& (\xi-1)(F U+T \Omega) \geqslant \iiint \hat{\Gamma}_{\square}(\Pi \square)-\left(F^{\square} U+T^{\square} \Omega\right) \\
& -(\delta-\lambda) \iiint \Gamma\left(\mathrm{U}_{*}^{\mathrm{V}}\right) d V \\
& -(\xi-\lambda)\left[\iiint \hat{\Gamma}\left(\Pi_{z}^{\mathrm{V}}\right) d V-\left(F^{\mathrm{V}} U+T^{\forall} \Omega\right)\right]
\end{aligned}
$$

(2) when $\alpha \geqslant \gamma \geqslant \beta$ and $\delta \geqslant \lambda \geqslant \xi$,

$$
\begin{array}{r}
r(F U+T \Omega) \geqslant \iiint \Gamma_{\Delta}\left(\Pi_{\varepsilon}^{\Delta}\right) d V-(\alpha-\gamma) \iiint \Gamma\left(\Pi_{e}^{\forall}\right) d V \\
-(\beta-\gamma) \iiint \Gamma_{*}\left(\Pi_{*}^{*}\right) d V \\
(\lambda-1)(F U+T \Omega) \geqslant \iiint \hat{\Gamma}_{\square}\left(\Pi_{\tau}^{\square}\right) d V-\left(F^{\square} U+T^{\square} \Omega\right) \\
-(\delta-\lambda) \iiint_{\int} \Gamma\left(\Pi_{\varepsilon}^{\mathrm{V}}\right) d V-(\xi-\lambda) \iiint \Gamma_{*}\left(\Pi_{*}^{*}\right) d V
\end{array}
$$

(3) when $\beta \geqslant \gamma \geqslant \alpha$ and $\xi \geqslant \lambda \geqslant \delta$,

$$
\begin{aligned}
& (\alpha+\beta-\gamma)(F U+T \Omega) \geqslant \iiint \Gamma_{\triangle}\left(\Pi_{\varepsilon}\right) d V \\
& -(\alpha-\gamma)\left[\iiint \hat{\Gamma}_{\circ}\left(\Pi^{\circ}\right) d V-\left(F^{\circ} U+T^{\circ} \Omega\right)\right] \\
& -(\beta-\gamma)\left[\iiint \hat{\Gamma}\left(\Pi_{\tau}^{\mathrm{V}}\right) d V-\left(F^{\mathrm{V} U}+T^{\mathrm{V}} \Omega\right)\right] \\
& (\delta+\xi-\lambda-1)(F U+T \Omega) \geqslant \iiint \hat{\Gamma}_{\square}\left(\Pi^{\square}\right) d V \\
& -\left(F^{\square} U+T^{\square} \Omega\right)-(\delta-\lambda)\left[\iiint \hat{\Gamma}_{\circ}\left(\Pi_{\tau}^{\circ}\right) d V\right. \\
& \left.-\left(F^{\circ} U+T^{\circ} \Omega\right)\right]-(\xi-\lambda)\left[\iiint \hat{\Gamma}\left(\Pi_{\tau}^{\mathrm{V}}\right) d V\right. \\
& \left.-\left(F^{\mathrm{V}} U+T^{\mathrm{V}} \Omega\right)\right]
\end{aligned}
$$

(4) when $\alpha, \beta \leqslant \gamma$ and $\delta, \xi \leqslant \lambda$,

$$
\begin{aligned}
& \alpha(F U+T \Omega) \geqslant \iiint \Gamma_{\triangle}\left(\Pi_{\odot}^{\triangle}\right) d V-(\alpha-\gamma)\left[\iiint \hat{\Gamma}_{\circ}\left(\Pi_{\tau}^{\circ}\right) d V\right. \\
& \left.-\left(F^{\circ} U+T^{\circ} \Omega\right)\right]-\left(\beta-\gamma \iiint \int \Gamma_{*}\left(\Pi_{\varepsilon}^{*}\right) d V\right. \\
& (\delta-1)(F U+T \Omega) \geqslant \iiint \hat{\Gamma}_{\square}\left(\Pi_{\tau}^{\square}\right) d V-\left(F^{\square} U+T^{\square} \Omega\right) \\
& -(\delta-\lambda)\left[\iiint \hat{\Gamma}_{\circ}\left(\Pi_{\tau}^{\circ}\right) d V-\left(F^{\circ} U+T^{\circ} \Omega\right)\right] \\
& -(\xi-\lambda) \iiint \Gamma_{*}\left(\Pi_{\varepsilon}^{*}\right) d V
\end{aligned}
$$

(5)

$$
F U+T \Omega \leqslant \iiint \Gamma\left(\Pi_{\varepsilon}^{\mathrm{V}}\right) d V-\iiint \hat{\Gamma}_{\circ}\left(\Pi^{\circ}\right) d V+\left(F^{\circ} U+T^{\circ} \Omega\right)
$$

$F U+T \Omega \geqslant \iiint \Gamma_{*}\left(\Pi_{*}^{*}\right) d V-\iiint \hat{\Gamma}\left(\Pi_{\tau}^{\mathrm{V}}\right) d V+\left(F^{\mathrm{V}} U+T^{\mathrm{V}} \Omega\right)$

[B] For case (b), the extremum princip les described by Eqs.(12) and (13) are used repeatedly in the following way for the fluid given earlier. That is,

$$
\begin{aligned}
& \iiint \Gamma\left(\Pi_{\varepsilon}^{V}\right) d V-\left(F U^{V}+T \Omega^{V}\right) \geqslant \iiint \Gamma\left(I_{\varepsilon}\right) d V \\
& -\iiint \hat{\Gamma}\left(\Pi_{\tau}\right) d V \geqslant-\iiint \hat{\Gamma}\left(\mathrm{II}_{\tau}^{\mathrm{V}}\right) d V \\
& -(F U+T \Omega) \\
& \iiint \Gamma_{*}\left(\Pi_{\varepsilon}\right) d V-(F U+T \Omega) \geqslant \iiint \Gamma_{*}\left(\amalg_{\varepsilon}^{*}\right) d V \\
& -\iiint \hat{\Gamma}_{0}\left(\Pi_{\tau}^{\circ}\right) d V \geqslant-\iiint \hat{\Gamma}_{0}\left(\Pi_{\tau}\right) d V \\
& -\left(F U^{*}+T \Omega^{*}\right) \\
& \iiint \Gamma_{\Delta}\left(\Pi_{\theta}\right) d V-(F U+T \Omega) \geqslant \iiint \Gamma_{\Delta}\left(\Pi_{\theta} \Delta\right) d V \\
& -\left(F U^{\nabla}+T \Omega^{\triangle}\right)
\end{aligned}
$$

Then the following relations are obtained from them: (1) when $\alpha, \beta \geqslant \gamma$ and $\delta, \xi \geqslant \lambda$,

$$
\begin{aligned}
& (\alpha-1)(F U+T \Omega) \geqslant \iiint \Gamma_{\triangle}\left(\Pi_{\ominus}^{\triangle}\right) d V-\left(F U^{\Delta}+T \Omega^{\Delta}\right) \\
& -(\alpha-\gamma)\left[\iiint \Gamma\left(\Pi_{e}^{\forall}\right) d V-\left(F U^{\mathrm{V}}+T \Omega^{\mathrm{V}}\right)\right] \\
& -(\beta-\gamma) \iiint \hat{\Gamma}\left(\Pi_{\tau}^{\forall}\right) d V
\end{aligned}
$$

$\delta(F U+T \Omega) \geqslant \iiint \hat{\Gamma}_{\square}\left(\Pi_{\tau}^{\square}\right) d V-(\delta-\gamma)\left[\iiint \Gamma\left(\Pi_{\varepsilon}^{\mathrm{V}}\right) d V\right.$

$$
\left.-\left(F U^{\mathrm{V}}+T \Omega^{V}\right)\right]-(\xi-\lambda) \iiint \hat{\Gamma}\left(\Pi_{z}^{V}\right) d V
$$

(2) when $\alpha \geqslant \gamma \geqslant \beta$ and $\delta \geqslant \lambda \geqslant \xi$,

$$
\begin{aligned}
& (\alpha+\beta-\gamma-1)(F U+T \Omega) \geqslant \iiint \Gamma_{\triangle}\left(\Pi_{\varepsilon}\right) d V \\
& -\left(F U^{\Delta}+T \Omega^{\triangle}\right)-(\alpha-\gamma)\left[\iiint \Gamma\left(\Pi_{s}^{V}\right) d V\right. \\
& \left.-\left(F U^{\mathrm{V}}+T \Omega^{\mathrm{V}}\right)\right]-(\beta-\gamma)\left[\iiint \Gamma_{*}\left(\Pi_{\varepsilon}^{*}\right) d V\right. \\
& \left.-\left(F U^{*}+T \Omega^{*}\right)\right] \\
& (\delta+\xi-\lambda)(F U+T \Omega) \geqslant \iiint \hat{\Gamma}_{\square}(\Pi \square) d V \\
& -(\delta-\lambda)\left[\iiint \Gamma\left(\Pi_{e}^{\mathrm{V}}\right) d V-\left(F U^{\mathrm{V}}+T \Omega^{\mathrm{V}}\right)\right] \\
& -(\xi-\lambda)\left[\iiint \Gamma_{*}\left(\Pi_{*}^{*}\right) d V-\left(F U^{*}+T \Omega^{*}\right)\right]
\end{aligned}
$$

(3) when $\beta \geqslant \gamma \geqslant \alpha$ and $\xi \geqslant \lambda \geqslant \delta$,

$$
\begin{gathered}
(\gamma-1)(F U+T \Omega) \geqslant \iiint \Gamma_{\triangle}\left(\Pi_{\varepsilon}^{\Delta}\right) d V-\left(F U^{\triangle}+T \Omega^{\Delta}\right) \\
-(\alpha-\gamma) \iiint \hat{\Gamma}_{0}\left(\Pi_{\tau}^{O}\right) d V-(\beta-\gamma) \iiint \hat{\Gamma}\left(\Pi_{t}^{V}\right) d V
\end{gathered}
$$

$\lambda(F U+T \Omega) \geqslant \iiint \hat{\Gamma}_{\square}\left(\Pi_{\tau}^{\square}\right) d V-(\delta-\lambda) \iiint \hat{\Gamma}_{\mathrm{o}}\left(\Pi_{\tau}^{\circ}\right) d V$

$-(\xi-\lambda) \iiint \hat{\Gamma}\left(\Pi_{z}^{V}\right) d V$

(4) when $\alpha, \beta \leqslant \gamma$ and $\delta, \xi \leqslant \lambda$,

$$
\begin{aligned}
& (\beta-1)(F U+T \Omega) \geqslant \iiint \Gamma_{\triangle}\left(\Pi_{\ominus}^{\Delta}\right) d V-\left(F U^{\Delta}+T \Omega^{\Delta}\right) \\
& -(\alpha-\gamma) \iiint \hat{\Gamma}_{\mathrm{\circ}}\left(\Pi_{\tau}^{\circ}\right) d V-(\beta-\gamma)\left[\iiint \Gamma_{*}\left(\Pi_{*}^{*}\right) d V\right. \\
& \left.-\left(F U^{*}+T \Omega^{*}\right)\right]
\end{aligned}
$$

$\xi(F U+T \Omega) \geqslant \iiint \hat{\Gamma}_{\square}\left(\Pi_{\tau}^{\square}\right) d V$

$$
\begin{aligned}
& -(\delta-\lambda) \iiint \hat{\Gamma}_{0}\left(\Pi_{\tau}^{\circ}\right) d V-(\xi-\lambda)\left[\iiint \Gamma_{*}\left(\Pi_{\varepsilon}^{*}\right) d V\right. \\
& \left.-\left(F U^{*}+T \Omega^{*}\right)\right]
\end{aligned}
$$

(5)

$$
\begin{aligned}
F U+T \Omega \geqslant \iiint \hat{\Gamma}_{0}\left(\Pi_{\tau}^{\circ}\right) d V-\iiint \Gamma\left(\Pi_{s}^{\mathrm{V}}\right) d V \\
+\left(F U^{\mathrm{V}}+T \Omega^{\mathrm{V}}\right)
\end{aligned}
$$


Fig. 1 Flow curves
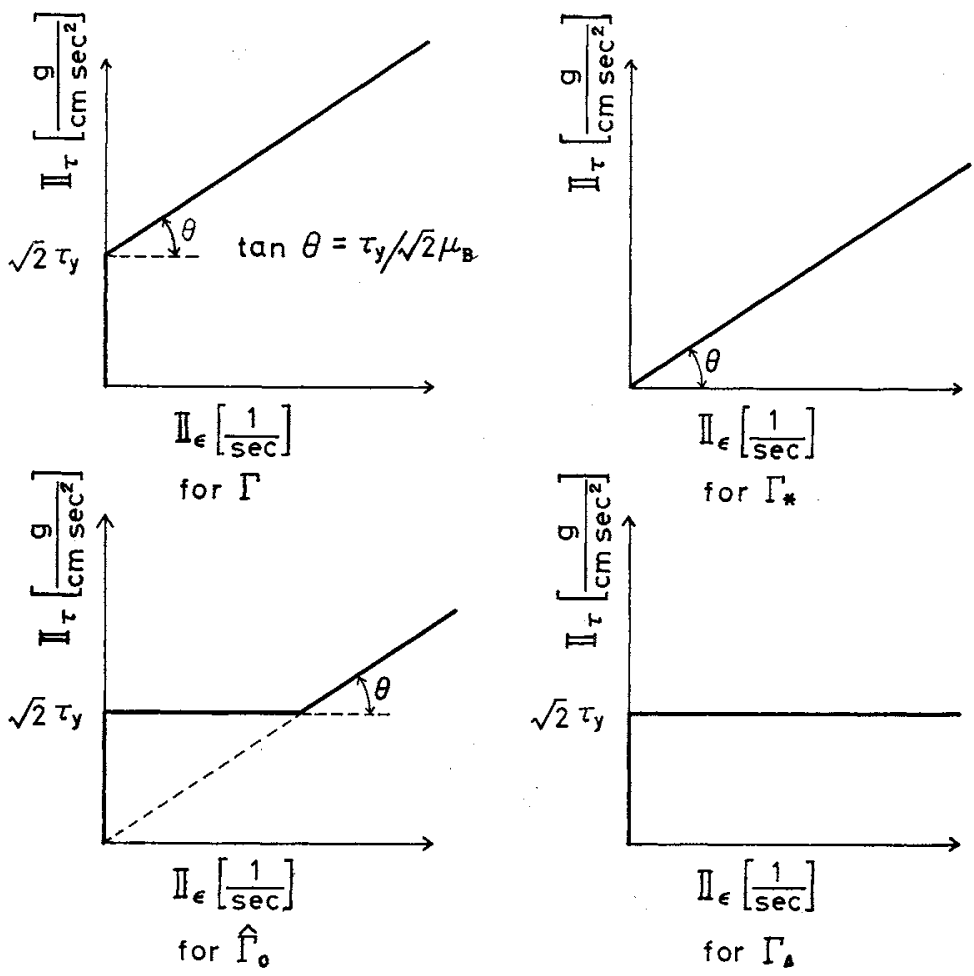

$$
\begin{gathered}
F U+T \Omega \geqslant \iiint_{\Gamma} \hat{\Gamma}\left(\Pi_{\tau}^{\forall}\right) d V-\iiint_{*} \Gamma_{*}\left(\Pi_{\varepsilon}^{*}\right) d V \\
+\left(F U^{*}+T \Omega^{*}\right)
\end{gathered}
$$

In the above expressions $\Pi_{\varepsilon}$, $\Pi_{\varepsilon}^{*}$, and $\Pi_{\varepsilon}^{\Delta}$ are derived from unique solution fields of the boundary value problems for the fluids with work functions $\Gamma, \Gamma_{*}$ and $\Gamma_{\triangle}$, respectively, and $\Pi_{*}^{\forall}$ from any kinematically admissible function $\mathscr{Y}_{i}^{V}$ for the related boundary value problem. Moreover, $\Pi_{\tau}, \Pi_{\tau}^{\circ}$ and $\Pi_{\tau}^{\square}$ are derived from unique stress fields of the boundary value problems for the fluids with complementary functions $\hat{\Gamma}$, $\hat{\Gamma}_{\circ}$ and $\hat{\Gamma} \square$, respectively, and $\Pi_{\tau}^{\forall}$ from any statically admissible function $\sigma_{i j}^{\forall}$ for the related boundary value problem.

For illustration let a Bingham fluid be considered. The constitutive equations are

$$
\begin{aligned}
& \tau_{i j}=2\left(\mu_{B}+\frac{\tau_{y}}{\sqrt{2} \mathrm{II}_{s}}\right) \varepsilon_{i j} \text { for } \mathrm{II}_{\tau} \geqslant \sqrt{2} \tau_{y} \\
& \varepsilon_{i j}=0 \text { for } \mathrm{I}_{\tau} \leqslant \tau_{y}
\end{aligned}
$$

and the invariant representation is

$$
\begin{aligned}
& \Pi_{\tau}=2 \mu_{B} \Pi_{\varepsilon}+\sqrt{2} \tau_{y} \text { for } \Pi_{\tau} \geqslant \sqrt{2} \tau_{y} \\
& \Pi_{\varepsilon}=0 \text { for } \Pi_{\tau} \leqslant \sqrt{2} \tau_{y}
\end{aligned}
$$

In consequence the work function and complementary function are as follows:

$$
\begin{aligned}
\Gamma\left(\Pi_{\varepsilon}\right) & =\mu_{B} \Pi_{\varepsilon}^{2}+\sqrt{2} \tau_{y} \Pi_{\varepsilon} \text { for } \Pi_{\tau} \geqslant \sqrt{2} \tau_{y} ; \\
& =0 \text { for } \Pi_{\tau} \leqslant \sqrt{2} \tau_{y} \\
\hat{\Gamma}\left(\Pi_{\tau}\right) & =\left(\Pi_{\tau}-\sqrt{2} \tau_{y}\right)^{2} / 4 \mu_{B} \text { for } \Pi_{\tau} \geqslant \sqrt{2} \tau_{y} ; \\
& =0 \text { for } \Pi_{\tau} \leqslant \sqrt{2} \tau_{y}
\end{aligned}
$$

Then the work and complementary functions for the new materials derivable from Eqs. (18) and (19) are

$$
\Gamma_{*}\left(\Pi_{\varepsilon}\right)=\mu_{B} \Pi_{s}^{2} \quad \text { for } \quad \Pi_{\varepsilon} \geqslant \sqrt{2} \tau_{y} ;
$$

$$
\begin{aligned}
& =0 \text { for } \Pi_{\tau} \leqslant \sqrt{2} \tau_{y} \\
\hat{\Gamma}_{\mathrm{o}}\left(\Pi_{\tau}\right) & =\left(\Pi_{\tau}^{2}-2 \tau_{y}^{2}\right) / 4 \mu_{B} \text { for } \Pi_{\tau} \geqslant \sqrt{2} \tau_{y} ; \\
& =0 \text { for } \Pi_{\tau} \leqslant \sqrt{2} \tau_{y}
\end{aligned}
$$

and for $\alpha=\delta=1$ and $\beta=\xi=-1$, Eqs. (20) and (21) become

$$
\begin{aligned}
\Gamma_{\triangle}\left(\mathrm{II}_{\varepsilon}\right) & =\sqrt{2} \tau_{y} \Pi_{\varepsilon} \text { for } \Pi_{\tau} \geqslant \sqrt{2} \tau_{y} ; \\
& =0 \text { for } \Pi_{\tau} \leqslant \sqrt{2} \tau_{y} \\
\hat{\Gamma}_{\square}\left(\Pi_{\tau}\right) & =\tau_{y}\left(\Pi_{\tau}-\sqrt{2} \tau_{y}\right) / \sqrt{2} \mu_{B} \text { for } \Pi_{\tau} \geqslant \sqrt{2} \tau ; \\
& =0 \text { for } \Pi_{\tau} \leqslant \sqrt{2} \tau_{y}
\end{aligned}
$$

The flow curves for these fluids are shown in Fig. 1. The material for $\Gamma_{*}$ is a Newtonian fluid with viscosity $\mu_{B}$, and that for $\Gamma_{\triangle}$ is a Mises solid, and that for $\hat{\Gamma}_{\mathrm{O}}$ is similar to the hypothetical fluid by Slattery ${ }^{6}$.

Now the inequality (33) gives under-estimates of the drag for a body moving in a Bingham fluid.

$$
F U \geqslant \iiint \Gamma_{*}\left(\Pi_{\varepsilon}^{*}\right) d V-\iiint \hat{\Gamma}\left(\amalg_{\tau}^{V}\right) d V+\iint T_{i}^{V} \mathscr{P _ { i }} d S_{v}
$$

The concrete analysis and results have already been reported $^{10)}$. All of the discussions in this section can also be applied to a pipeline flow problem.

\section{Conclusions}

From this analysis the following conclusions can be deduced:

(1) The important inequality Eq. (9) can be obtained from the extremum principles only if the flow is of no body forces and no surface of velocity discontinuity. (2) The common value of the functionals in the actual state gives the total rate of dissipation of energy only if the fluid is a power model, and then the important equations (16) and (17) can be deduced. 
(3) The important qualitative propositions for a Newtonian fluid given by Hill have been extended to deductions (1) to (5) in Sect. 2 for a power model. (4) The important inequalities Eqs.(24) to (43), giving over-estimates or under-estimates for a physical unknown, have been obtained.

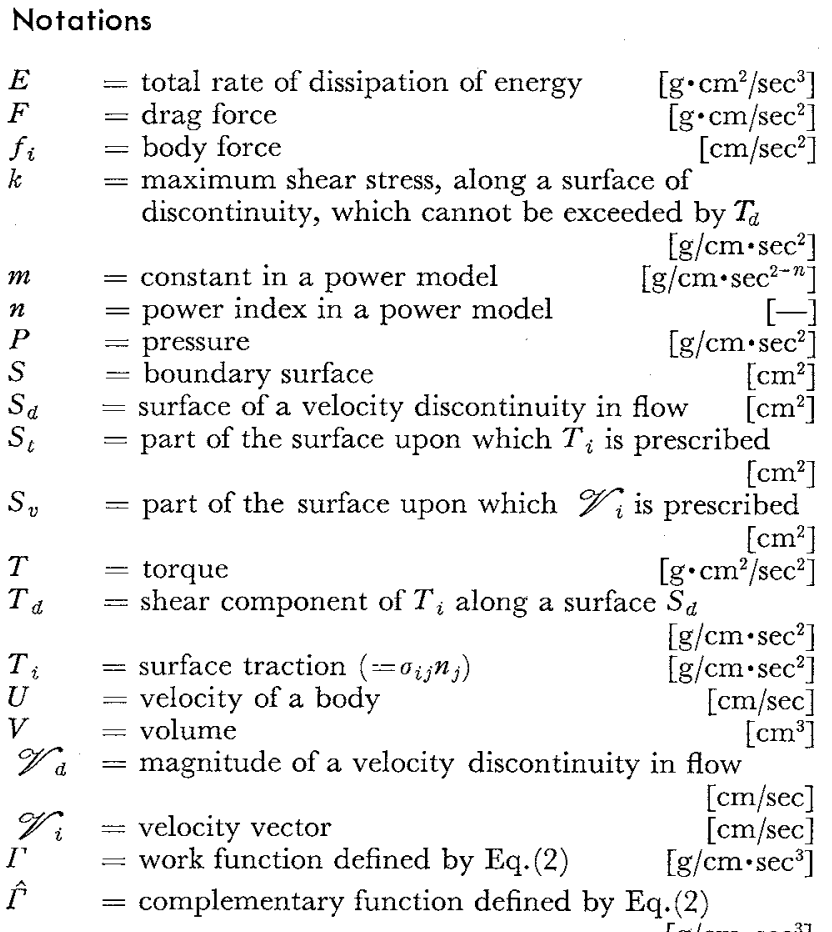

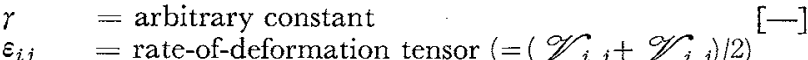

$[1 / \mathrm{sec}]$

$\begin{aligned} \eta, \hat{\eta} & =\text { coefficients defined by Eq. }(1) \quad[\mathrm{g} / \mathrm{cm} \cdot \mathrm{sec}]\end{aligned}$

$\begin{array}{ll}\lambda & =\text { arbitrary constant } \\ \mu_{B} & =\text { coefficient of viscosity of a Bingham fluid }\end{array}$

$[\mathrm{g} / \mathrm{cm} \cdot \mathrm{sec}]$

$\rho \quad=$ density

$\tau_{i j} \quad=$ deviation stress tensor

$\tau_{y} \quad=$ yield stress of a Bingham fluid

$\Omega \quad=$ angular veloctiy

[1/sec] $\left[\mathrm{g} / \mathrm{cm}^{3}\right]$ tensor and of the stress tensor defined by Eq.(3)

〈Superscripts〉

$\left[\mathrm{g} / \mathrm{cm} \cdot \mathrm{sec}^{2}\right],[1 / \mathrm{sec}]$

$+\quad=$ derived from a trial velocity function

$0=$ derived from a trial stress function

\section{References}

1) Haddow, J. B. and H. Luming: Appl. Sci. Res., A15, $81(1965)$

Haddow, J. B. and H. Luming: Appl. Sci. Res., A16, 469 (1966)

2) Hill, R.: J. Mech. Phy. Solids, 5, 66 (1956)

3) Hill, R. and G. Power: Quart. J. Mech. Appl. Math., 9, 313 (1965)

4) Ito, E.: Kikai Gakkai Ronbunshū, 17, 130 (1951)

5) Schechter, R. S.: A.I.Ch.E. Journal, 7, 445 (1961)

6) Slattery, J. C.: Appl. Sci. Res., A10, 286 (1960)

7) Tomita, Y.: Bull. Japan Soc. Mech. Engrs., 2, 469 (1959)

8) Wasserman, M. L. and J. C. Slattery: A.I.Ch.E. Journal, 10, 383 (1964)

9) Yoshioka, N. and K. Adachi: J. Chem. Eng. Japan, 4, 217 (1971)

10) Yoshioka, N., K. Adachi and H. Ishimura: Preprint for Chem. Eng. Meeting at Yonezawa (1970)

\title{
CONTRIBUTION OF SOLIDS DISPERSED IN BUBBLES TO MASS TRANSFER IN FLUIDIZED BED*
}

TETSU WAKABAYASHI AND DAIZO KUNII

Department of Chemical Engineering, University of Tokyo, Tokyo, Japan

\begin{abstract}
Experimental studies were carried out in a gas-solid fluidized bed with a diameter of $20 \mathrm{~cm}$, into which highly humid air was sent to form successive single bubbles larger than $4 \mathrm{~cm}$ by use of a solenoid valve.

Overall rate constant of mass transfer between single bubble and solids in emulsion was found to decrease with increasing bubble diameter. Comparison of the above experimental data with a theoretical equation proposed by Kunii and Levenspiel taking account of the solids dispersed in bubble showed that the contribution of the dispersed solids was nearly controlling and that most of the mass transfer between bubble and solids in the above fluidized bed was carried out by solids dispersed in bubble, even though the volume fraction of the dispersed solids to the bubble was as small as $4 \times 10^{-4}$.
\end{abstract}

\section{Introduction}

Recently three groups of investigators $\left.{ }^{2}, 3,8\right)$ using different experimental techniques independently found

* Received on September 18, 1970 that solids are present in bubbles in fluidized beds. However, nothing so far has been said about the influence of the dispersed solids on the transfer rates between gas and solids. This paper aims to shed light on the above problem. 